\title{
A Note on Self-Accelerating Olver and Olver-Gauss Beams
}

\section{Ioannis M. Besieris ${ }^{*}$, Amr M. Shaarawi², Bradley Davis ${ }^{3}$}

${ }^{1}$ The Bradley Department of Electrical and Computer Engineering, Virginia Polytechnic Institute and State University, Blacksburg, VA, USA

${ }^{2}$ Department of Physics, The American University in Cairo, New Cairo, Egypt

${ }^{3}$ Ted and Karyn Hume Center for National Security and Technology, Virginia Polytechnic Institute and State University, Blacksburg, VA, USA

Email: ×besieris@vt.edu, shaarawi@aucegypt.edu, brdavis@vt.edu

How to cite this paper: Besieris, I.M., Shaarawi, A.M. and Davis, B. (2019) A Note on Self-Accelerating Olver and Olver-Gauss Beams. Optics and Photonics Journal, 9, 1-7.

https://doi.org/10.4236/opj.2019.91001

Received: December 12, 2018

Accepted: January 14, 2019

Published: January 17, 2019

Copyright (c) 2019 by author(s) and Scientific Research Publishing Inc. This work is licensed under the Creative Commons Attribution International License (CC BY 4.0).

http://creativecommons.org/licenses/by/4.0/

\begin{abstract}
The recent introduction by Belafhal et al. [Opt. and Photon. J. 5, 234-246 (2015)] of $m$ th-order Olver beams as a novel class of self-accelerating nondiffracting solutions to the paraxial equation is a direct contradiction to the seminal work of Berry and Balazs who determined that the infinite-energy Airy wave packet is the only accelerating nondiffracting solution to the $(1+1) \mathrm{D}$ Schrödinger equation. It is shown in this note that the work of Belafhal et al. is valid only for $m=0$, which coincides with the Airy solution.
\end{abstract}

\section{Keywords}

Nondiffracting Waves, Nonlinearly Self-Bending Beams, Airy Beam, Accelerating Olver and Olver-Gauss Beams

\section{Introduction}

Extensive studies of self-accelerating beams have been made recently. The basic such beam is the Airy solution

$$
\psi(x, z)=A i\left[x-\left(\frac{z}{2}\right)^{2}\right] \exp \left[-i\left(\frac{z^{3}}{12}-\frac{x z}{2}\right)\right]
$$

governed by the paraxial equation

$$
i \frac{\partial}{\partial z} \psi(x, z)+\frac{1}{2} \frac{\partial^{2}}{\partial x^{2}} \psi(x, z)=0
$$

in free space. Here, $x=X / X_{0}$ and $z=Z /\left(k X_{0}^{2}\right)$ are, respectively, dimensionless transverse and longitudinal variables, defined in terms of the original 
variables $X$ and $Z$, the wavenumber $k$, and a scaling factor $X_{0}$ with units of meters. The complexification $z \rightarrow z-i 2 a_{1}$, where $a_{1}$ is a positive parameter, ensures the square integrability (finite energy) of the input function $\psi(x, 0)$ and, hence, of $\psi(x, z)$ for $z>0$. The finite-energy version of the solution given in Equation (1) was first formulated analytically by Siviloglou and Christodoulides [1] and subsequently demonstrated experimentally by Siviloglou et al. [2]. Their work was motivated by the infinite-energy (nonspreading) accelerating Airy solution to the Schrödinger equation introduced by Berry and Balazs [3] in the context of quantum mechanics. A full wave theoretical analysis of the Airy beam has been undertaken by Kaganovsky and Heyman [4]. An Airy beam is slowly diffracting; it can retain its intensity over several diffraction lengths while bending laterally along a parabolic path even though its centroid is constant. Another feature, which has been demonstrated both analytically and experimentally, is that an Airy beam propagating in free space can perform ballistic dynamics akin to those of projectiles moving under the action of gravity.

Both bending Airy beams and accelerating Airy wavepackets are characterized by self-healing properties; they tend to reform in spite of the severity of imposed perturbations; this is due to the reinforcement of the main lobe by the side lobes. The robustness of such beams has been studied in the presence of material dispersion [5], deterministic inhomogeneities (see [6] and references therein), and turbulent environments [7] [8]. These exotic properties suggest various physical applications, such as Airy beam-mediated particle cleaning and vacuum electron acceleration. A recent review of the theory, generation and applications of Airy beams has been published by $\mathrm{Hu}$ et al. [9].

\section{Critical Comments on Accelerating Olver Beams}

Belafhal et al. [10] have reported a new class of "accelerating" (more precisely, nonlinearly self-bending) nondiffracting solutions of the free-space parabolic equation [cf. Equation (2)] in terms of the Olver functions

$$
O_{m}(x)=\frac{1}{2 \pi} \int_{-\infty}^{\infty} \mathrm{d} \lambda \exp \left[a(i \lambda)^{\gamma}+i \lambda x\right] ; \gamma=m+3,|a|=\frac{1}{m+3},
$$

which, in turn, are related to the solutions of the ordinary differential equation

$$
\frac{\mathrm{d}^{2}}{\mathrm{~d} x^{2}} W_{\mp}(x) \mp \frac{1}{4} n^{2} x^{n-2} W_{\mp}(x)=0 ; n>2 .
$$

Specifically, the authors state that a novel class of nondiffracting solutions to Equation (2) is given as follows:

$$
\begin{aligned}
\psi_{m}(x, z) & =\frac{1}{2 \pi} \int_{-\infty}^{\infty} \mathrm{d} \lambda \exp \left[a(i \lambda)^{m+3}-i \frac{1}{2} \lambda^{2} z+i \lambda x\right] \\
& =\exp \left[i\left(\frac{z x}{2}-\frac{z^{3}}{12}\right)\right] O_{m}\left(x-\frac{z^{2}}{4}\right) ;|a|=\frac{1}{m+3} .
\end{aligned}
$$

The first equality above is correct. The integral expression does, indeed, satisfy the parabolic Equation (2) for all values of $m$, as the authors have shown in 
Appendix B of Ref. [10]. However, the second equality above is incorrect. It holds only for $m=0$, which yields the well-known Airy beam solution given in Equation (1). This affects the statements in Equations (3) and (4), Equation (A-1) and Equations (B-6) and (B-7) in Ref. [10]. A general proof of our assertion can be provided by using the ansatz

$$
\psi(x, z)=\exp \left[i\left(\frac{x z}{2}-\frac{s^{3}}{12}\right)\right] W\left(x-\frac{z^{2}}{4}\right)
$$

into the parabolic Equation (2). As a result, one obtains the ordinary differential equation

$$
\frac{\mathrm{d}^{2}}{\mathrm{~d} \xi^{2}} W(\xi)-\xi W(\xi)=0 ; \xi=x-\frac{s^{2}}{4},
$$

a solution of which is the Airy function; specifically, $W(\xi)=A i(\xi)=2 \pi O_{0}(\xi)$. This is in contradiction to the second equality in Equation (5) in which the Olver functions are related to the solutions to Equation (4) for $m>0$.

The authors give in Equation (24) of Ref. [10] the spectra of exponentially apodized initial conditions $\psi_{m}(x, 0)$ as follows:

$$
\psi_{m}(x, 0)=O_{m}(x) \exp (a x) \leftrightarrow \tilde{O}_{m}(\lambda+i a),
$$

for $m=0,1$ and 2. Here, $\tilde{O}_{m}(\lambda)$ denotes the Fourier transform of the Olver function $O_{m}(x)$. For $z \geq 0$, one obtains, then,

$$
\begin{aligned}
& \psi_{0}(x, z)=\exp \left(a x+i \frac{a^{2}}{2} z\right) \int_{-\infty}^{\infty} \mathrm{d} \lambda \exp [i \lambda(x+i a z)] \exp \left(-i \frac{\lambda^{2}}{2} z\right) \exp \left(i \frac{\lambda^{3}}{3}\right), \\
& \psi_{1}(x, z)=\exp \left(a x+i \frac{a^{2}}{2} z\right) \int_{-\infty}^{\infty} \mathrm{d} \lambda \exp [i \lambda(x+i a z)] \exp \left(-i \frac{\lambda^{2}}{2} z\right) \exp \left(-\frac{\lambda^{4}}{4}\right), \\
& \psi_{2}(x, z)=\exp \left(a x+i \frac{a^{2}}{2} z\right) \int_{-\infty}^{\infty} \mathrm{d} \lambda \exp [i \lambda(x+i a z)] \exp \left(-i \frac{\lambda^{2}}{2} z\right) \exp \left(i \frac{\lambda^{5}}{5}\right) .
\end{aligned}
$$

The wave function $\psi_{0}(x, z)$ is the well-known Airy beam and contains finite energy for a positive parameter $a$. The Fourier representations of the wavefunctions $\psi_{m}(x, z)$ given by the integral expressions above do not yield analytical solutions for $m>0$. The expressions for $m=1$ and 2 could justifiably be called Olver beams (but not necessarily accelerating Olver beams) because they are associated with apodized fourth-order diffusion (super diffusion) and fifth-order Airy (hyper Airy) functions, respectively, at $z=0$. However, a careful examination is needed in order to establish whether the exponential apodization is sufficient for ensuring finite energy. It turns out that the Airy beam $\psi_{1}(x, z)$ contains finite energy only for $a<0$. The choice of appropriate apodization for $\psi_{2}(x, z)$ requires careful examination.

Due to their complexity, the wave functions $\psi_{m}(x, z)$ for $m=1$ and 2 can be examined only numerically. The authors allude to such a program in Sec. 4, where they propose hologram masks. However, it is not clear whether the integrations given in Equation (9) above, which are required for $z>0$, have been carried out, and what is the basis for the plots in Figure 3 of Ref. [10]. Why do 
the plots for the fourth-order $(m=1)$ and sixth-order $(m=3)$ super diffusion, characterized essentially by single lobes, appear multi-lobed under finite-energy (apodised) conditions?

Consider the second expression in Equation (5), viz.,

$$
\psi_{m}(x, z)=\exp \left[i\left(\frac{z x}{2}-\frac{z^{3}}{12}\right)\right] O_{m}\left(x-\frac{z^{2}}{4}\right) .
$$

It has been shown in this note that this does not represent a solution of the parabolic Equation (2), unless $m=0$. Is it possible, however, that there exists another type of equation the solutions of which can be expressed as in Equation (10) for all values of $m$ ? Such an equation does exist and it is given as follows:

$$
\left[i \frac{\partial}{\partial z}+\frac{1}{2} \frac{\partial^{2}}{\partial x^{2}}+\frac{1}{2}\left(x-\frac{z^{2}}{4}\right)-\frac{1}{8} m^{2}\left(x-\frac{z^{2}}{4}\right)^{m-2}\right] \psi_{m}(x, z)=0 .
$$

Introducing the ansatz given in Equation (6) into Equation (11), one obtains the ordinary differential equation

$$
\frac{\mathrm{d}^{2}}{\mathrm{~d} \xi^{2}} W_{m}(\xi)-\frac{1}{4} m^{2} \xi^{m-2} W_{m}(\xi)=0 ; \xi=x-\frac{z^{2}}{4},
$$

which has been studied extensively by Olver [11]. The solutions $W_{m}(\xi)$ are related to the Olver functions; for example,

$$
W_{3}(\xi)=O_{0}\left[(3 / 2)^{2 / 3} \xi\right] .
$$

Since Equation (12) above is identical to Equation (1) in Ref. [10], with $f(t)$ given in Equation (6), the specific result in Equation (13) above indicates that Equation (6) in [10] is invalid.

\section{Critical Comments on Accelerating Olver-Gauss Beams}

Hennani et al. [12] have used the Fresnel diffraction formula for a paraxial ABCD optical system, viz.,

$$
U_{2}\left(x_{2}\right)=\left(\frac{i k}{2 \pi B}\right)^{1 / 2} \int_{-\infty}^{\infty} \mathrm{d} x_{1} U_{1}\left(x_{1}\right) \exp \left[-\frac{i k}{2 B}\left(A x_{1}^{2}-2 x_{1} x_{1}+D x_{2}^{2}\right)\right],
$$

in order to determine the output due to the input Olver-Gauss function

$$
U_{1}\left(x_{1}\right)=O_{n}\left(\frac{x_{1}}{\omega_{0}}\right) \exp \left(a_{0} \frac{x_{1}}{\omega_{0}}\right) \exp \left(-b_{0} \frac{x_{1}^{2}}{\omega_{0}^{2}}\right) .
$$

Here, $\omega_{0}$ is a normalization parameter with units of length, and $a_{0}, b_{0}$ are positive dimensionless parameters used to ensure finite energy. The main result is given in Equation (7) of Ref. [12], viz.,

$$
U_{2}\left(x_{2}\right)=\frac{1}{2 \pi}\left(\frac{i k}{2 \pi B}\right)^{1 / 2}\left(\frac{\pi}{\frac{i k A}{2 B}+\frac{b_{0}}{\omega_{0}^{2}}}\right)^{1 / 2} \exp \left(-\frac{i k D}{2 B} x_{2}^{2}\right) \exp \left[\frac{\left(\frac{a_{0}}{\omega_{0}}+\frac{i k}{b} x_{2}\right)^{2}}{4\left(\frac{i k A}{2 B}+\frac{b_{0}}{\omega_{0}^{2}}\right)}\right]^{2}
$$




$$
\begin{aligned}
& \times \exp \left[\frac{1}{96} \frac{1}{\omega_{0}^{6}\left(\frac{i k A}{2 B}+\frac{b_{0}}{\omega_{0}^{2}}\right)^{3}}\right] \exp \left[\frac{1}{8} \frac{\left(\frac{a_{0}}{\omega_{0}}+\frac{i k}{b} x_{2}\right)}{\omega_{0}^{3}\left(\frac{i k A}{2 B}+\frac{b_{0}}{\omega_{0}^{2}}\right)^{2}}\right] \\
& \times O_{n}\left[\frac{\frac{a_{0}}{\omega_{0}}+\frac{i k}{b} x_{2}}{2 \omega_{0}\left(\frac{i k A}{2 B}+\frac{b_{0}}{\omega_{0}^{2}}\right)}+\frac{1}{16 \omega_{0}^{4}\left(\frac{i k A}{2 B}+\frac{b_{0}}{\omega_{0}^{2}}\right)^{2}}\right] .
\end{aligned}
$$

Unfortunately, this result is incorrect, in general, because it is based on a variation of the expression given in Equation (5); specifically,

$$
\frac{1}{2 \pi} \int_{-\infty}^{\infty} \mathrm{d} \lambda \exp \left[a(i \lambda)^{n+3}-\frac{1}{2} \lambda^{2} s+i \lambda x\right]=\exp \left(\frac{s x}{2}+\frac{s^{3}}{12}\right) O_{n}\left(x+\frac{s^{2}}{4}\right) .
$$

This formula and, as consequence, Equation (16), is valid only for $n=0$. In this case, the solution in Equation (16) corresponds to a finite-energy accelerating Airy-Gauss beam which has been studied previously (see, e.g., Ez-Zariy et al. [13]). All the other cases examined analytically and numerically for different ABCD parameters in Ref. [12] are incorrect.

The free-space version of Equation (16) corresponding to the optical $A B C D$ matrix

$$
\left(\begin{array}{ll}
A & B \\
C & D
\end{array}\right)=\left(\begin{array}{ll}
1 & z \\
0 & 1
\end{array}\right)
$$

with $z$ the direction of propagation, has been used by Hennani et al. [14] in order to study the action of the radiation forces produced by a highly focused finite-energy Olver-Gaussian beam on a Rayleigh dielectric sphere. For the reasons detailed above, the numerical results presented by the authors are valid only for the case $n=0$.

\section{Concluding Remarks}

In the seminal work of Berry and Balazs [3] it was determined that the infinite-energy Airy wave packet is the only self-accelerating nondiffracting solution to the $(1+1)$ D Schrödinger equation. Within the framework of optics, Siviloglou and Christodoulides [1] rendered the Airy beam given in Equation (1) physically realizable by appropriate apodization of the initial wave function, viz., $\psi(x, 0)=A i(x) \mathrm{e}^{a_{1} x}$, with a positive parameter $a_{1}$.

The introduction by Belafhal et al. [10] of the Olver beams as a novel class of self-accelerating nondiffracting solutions to the paraxial Equation (2) is a direct contradiction to the work of Berry and Balazs. It has been shown in this note that the work in [10] is valid only for $m=0$, which coincides with the Airy solution. Unfortunately, the mistakes in [10] have been carried over to the work by Hennani et al. [12] on the propagation of Olver-Gauss functions through an optical ABCD system, and by Hennani et al. [14] on the action of the radiation 
forces produced by a highly focused finite-energy Olver-Gaussian beam on a Rayleigh dielectric sphere.

In closing, it should be pointed out that the Olver functions

$$
O_{m}(x)=\frac{1}{2 \pi} \int_{-\infty}^{\infty} \mathrm{d} \lambda \exp \left[a(i \lambda)^{\gamma}+i \lambda x\right] ; \gamma=m+3,|a|=\frac{1}{m+3},
$$

can be considered as "incomplete" elementary catastrophe integrals; the latter are defined as

$$
C_{n}(\vec{q})=\int_{R} \mathrm{~d} \lambda \exp \left[i P_{n}(\vec{q}, \lambda)\right] ; P_{n}(\vec{q}, \lambda)=\frac{\lambda^{n}}{n}+\sum_{j=1}^{n-2} q_{j} \frac{\lambda^{j}}{j} .
$$

$C_{3}(q=x)$ is precisely equal to $O_{0}(x)$ and corresponds to the fold catastrophe. $C_{n}(\vec{q})$ for $n=4,5$ and 6 are the cusp, swallowtail and butterfly catastrophes, respectively. The dynamics of the cusp, swallowtail and the butterfly optical catastrophes have been studied in terms of solutions to the 3D paraxial equation [15] [16] [17]. There exist more complicated catastrophe integrals than those in Equation (20); for example, the hyperbolic umbilic. Self-accelerating solutions of the 3D paraxial equation associated with this catastrophe have been determined recently [18] [19].

\section{Conflicts of Interest}

The authors declare no conflicts of interest regarding the publication of this paper.

\section{References}

[1] Siviloglou, G.A. and Christodoulides, D.N. (2007) Accelerating Finite Energy Airy Beams. Optics Letters, 32, 979-981. https://doi.org/10.1364/OL.32.000979

[2] Siviloglou, G.A., Broky, J., Dogariu, A. and Christodoulides, D.N. (2007) Observation of Accelerating Airy Beams. Physical Review Letters, 99, 213901. https://doi.org/10.1103/PhysRevLett.99.213901

[3] Berry, M.V. and Balazs, N.L. (1979) Nonspreading Wavepackets. American Journal of Physics, 47, 264-267.

[4] Kaganovsky, Y. and Heyman, E. (2010) Wave Analysis of Airy Beams. Optics EXpress, 18, 8440-8452. https://doi.org/10.1364/OE.18.008440

[5] Besieris, I.M. and Shaarawi, A.M. (2008) Accelerating Airy Wave Packets in the Presence of Quadratic and Cubic Dispersion. Physical Review E, 78, 046005. https://doi.org/10.1103/PhysRevE.78.046605

[6] Besieris, I.M., Shaarawi, A.M. and Zamboni-Rached, M. (2016) Accelerating Airy Beams in the Presence of Inhomogeneities. Optics Communications, 369, 56-64. https://doi.org/10.1016/j.optcom.2016.02.020

[7] Gu, Y. and Gbur, G. (2010) Scintillations of Airy Beam Arrays in Atmospheric Turbulence. Optics Letters, 35, 3456-3458. https://doi.org/10.1364/OL.35.003456

[8] Chu, X. (2011) Evolution of an Airy Beam in Turbulence. Optics Letters, 36, 2701-2703. https://doi.org/10.1364/OL.36.002701

[9] Hu, Y., Siviloglou, G.A., Zhang, P., Efremidis, N.K., Christodoulides, D.N. and Chen, Z. (2012) Self-Accelerating Airy Beams: Generation, Control and Applications. In: 
Chen, Z. and Marandotti, R., Eds., Nonlinear Photonics and Novel Optical Phenomena, Springer Series in Optical Sciences 170, New York, 1-44.

[10] Belafhal, A., Ez-Zariy, L., Hennani, S. and Nebdi, H. (2015) Theoretical Introduction and Generation Method of Novel Nondiffracting Waves: Olver Beams. Optics and Photonics Journal, 5, 234-246. https://doi.org/10.4236/opj.2015.57023

[11] Olver, F.W.J. (1977) Connection Formulas for Second-Order Differential Equations with Multiple Turning Points. SIAM Journal on Mathematical Analysis, 8, 127-154. https://doi.org/10.1137/0508009

[12] Hennani, S., Ez-Zariy, L. and Belafhal, A. (2015) Propagation of Finite Olver-Gaussian Beams Passing Through a Paraxial ABCD Optical System. Optics and Photonics Journal, 5, 273-294. https://doi.org/10.4236/opj.2015.59026

[13] Ez-Zariy, L., Hennani, S., Nebdi, H. and Belafhal, A. (2014) Airy-Gaussian Beams Passing Through a Misaligned Optical System with Finite Aperture. Optics and Photonics Journal, 4, 325-336. https://doi.org/10.4236/opj.2014.411033

[14] Hennani, S., Ez-Zariy, L. and Belafhal, A. (2015) Radiation Forces on a Dielectric Sphere Produced by Finite Olver-Gaussian Beams. Optics and Photonics Journal, 5, 344-353. https://doi.org/10.4236/opj.2015.512033

[15] Ren, Z.J., Ying, C.F., Jin, H.Z. and Chen, B. (2015) Generation of a Family of Pearcy Beams Based on Fresnel Diffraction Catastrophes. Journal of Optics, 17, 105608. https://doi.org/10.1088/2040-8978/17/10/105608

[16] Zannotti, A., Diebel, F. and Denz, C. (2017) Dynamics of the Optical Swallowtail Catastrophe. Optica, 4, 1157-1162. https://doi.org/10.1364/OPTICA.4.001157

[17] Zannotti, A., Diebel, F., Bogulawski, M. and Denz, C. (2017) Optical Catastrophes and the Swallowtail and Butterfly Beams. New Journal of Physics, 19, 053004. https://doi.org/10.1088/1367-2630/aa6ecd

[18] Berry, M.V. (2017) Stable and Unstable Airy-Related Caustics and Beams. Journal of Optics, 19, 055601. https://doi.org/10.1088/2040-8986/aa6281

[19] Ring, J.D. (2013) Incomplete Catastrophes and Paraxial Beams. PhD Thesis, University of Bristol, UK. 